\title{
Die Geschichte von den zwei Impeachments: Medien, Internet, Umfragen
}

Die Rolle der Medien - analog wie digital - ist seit der Kampagne um das Weiße Haus 2016 zentral für Donald Trump und seine Präsidentschaft, in guten wie in schlechten Zeiten. Die Erfahrung einer radikal polarisierten Öffentlichkeit hat der Präsident bereits während des Wahlkampfs erlebt; bei seinen campaign rallies traf er (fast) im ganzen Land auf ein elektrisiertes, enthusiastisches Publikum, das ihm überall hin folgen würde. Und in der medial vermittelten Öffentlichkeit sah er sich offener Häme ebenso wie harscher Kritik gegenüber, fand dort aber auch vorbehaltlose Unterstützung - größer konnten die Gegensätze nicht sein. Das Leben in geteilten Medienrealitäten wurde spätestens seit der Amtseinführung im Januar 2017 zu einer Standardsituation der Regierungskommunikation: „Wir gegen die“ oder „Die gegen uns“ waren die einzig gültigen Perspektiven auf die kommunikative Situation rund um das Weiße Haus. Wesentliche Beiträge dazu lieferten drei zentrale Säulen der öffentlichen Kommunikation: die Berichterstattung in den traditionellen Massenmedien (wahlweise als Tageszeitung oder klassisches Network TV), Status und Stimmung in den sozialen Netzwerken im Internet sowe die regelmäßigen Umfragen großer Meinungsforschungsinstitute.

Genau hier liegt ein zentraler Unterschied $\mathrm{zu}$ den bisherigen Impeachment-Verfahren. Über Andrew Jackson und die Ansätze einer bürgerlichen Öffentlichkeit im 19. Jahrhundert muss hier nicht weiter diskutiert werden wohl aber über die Situation Ende des 20. Jahrhunderts beim Impeachement gegen Bill Clinton. Zwar hatte hier mit Matt Drudge ein Online-Journalist die Dynamik der öffentlichen Debatte angeheizt, doch war der zentrale Impuls zur Skandalisierung der Situation um die Praktikantin Monica Lewinsky von traditionellen Medienakteuren ausgegangen. Auch hatte der Präsident vor allem auf das Fernsehen gesetzt, als es um eine Gegenstrategie ging: Das öffentliche 
Schuldeingeständnis wurde als Videobotschaft transportiert und ebnete schließlich den Weg zum Freispruch im Senat. Profitieren konnte Bill Clinton zudem von einer weniger harten Frontlinie zwischen den Parteien: Ein Konsens quer zu den Parteigrenzen war seinerzeit durchaus noch möglich.

Als im Spätsommer 1998 die republikanische Mehrheit im Repräsentantenhaus das Impeachment gegen Bill Clinton vorbereitete, stimmten 31 Demokraten mit den Republikanern - zur Erleichterung der Fraktionsführung der Demokraten, denn man hatte mit weit mehr gerechnet. Bei Trump wären wohl deutlich weniger „Abweichler“ bereits als dramatisch empfunden worden. Wenn noch ein Beleg notwendig gewesen wäre für die extreme Polarisierung Amerikas, dann hätte diese Abstimmung im November 2019 ihn erbracht: Dass nicht einer der 230 Republikaner*innen im Haus das Verfahren unterstützte, war symbolträchtig genug.

\section{Divided country, divided media}

Die eigentliche Bewertung des präsidentiellen „Verhaltens“ stand da längst hinter der Auseinandersetzung um die öffentliche Meinung zurück. Das konnte man erwarten: Schon bei Bill Clinton und Richard Nixon, in einem weit weniger gespaltenen Land, war es diese public opinion, die den Ausschlag gab. Sie zeigte den Protagonisten auf, wie ihre (Wieder-)Wahlchancen aussahen - im Spiegel der Meinungen ihrer Wahlkreise. Vielleicht sollte man angesichts dieser Parallelwelten besser von zwei Verfahren sprechen, die an zwei Publika gerichtet waren, durchaus im Stil einer direkten Wähleransprache in sorgfältig voneinander getrennten politischen Öffentlichkeiten und Lebenswelten.

Die Demokraten sahen sich „historisch verpflichtet“. Der Präsident habe nicht nur die amerikanischen Sicherheitsinteressen in der Ukraine aus persönlichen Motiven riskiert, er attackiere die Gewaltenteilung und damit die Verfassung höchstselbst. Dass es sich hier nicht nur um ein missverständliches Telefonat und ein Hörensagen gehandelt hat, sondern um eine rücksichtslose Schattendiplomatie, das hätten die öffentlichen Anhörungen deutlich gemacht.

Demgegenüber folgten die Republikaner im Kongress nahezu unisono Trumps Linie der totalen Obstruktion. Schließlich sei der Präsident nicht mit einer smoking gun erwischt worden. Die Vorwürfe beruhten allesamt auf Gerüchten, seien weder substanziell noch justitiabel. All die Geschichten, die da in langen Stunden der Anhörung um ein harmloses Telefonat kreisten, seien nichts weniger als eine Schmierenkomödie (und kein von der Verfassung geschütztes Verfahren). Eine solche Mentalität richtete sich natürlich an Trump selbst; aber auch an die 
Unterstützer des Präsidenten und zeigte ihnen, dass es nach wie vor gelte, den (sozialistischen) Demokraten zu misstrauen. Und es war erneut viel Lärm um den deep state. Dazu passte das Bild der frustrierten Demokraten nur allzu gut. Mehr noch: in den öffentlichen Anhörungen manifestierte sich dieser Schattenstaat überaus stimmig in den befragten Diplomaten.

\section{Vom Inside- zum Outside-Game}

Lebendig erhalten wurde diese deep-state-Erzählung vor allem von Fox News, Trumps medialem Rückhalt. Er lieferte den (möglicherweise zweifelnden) republikanischen Senatoren die Vorstellung einer ungetrübt dem Präsidenten folgenden Öffentlichkeit. Dabei bediente der Sender vornehmlich die Idee, die im Raum stehenden Vorwürfe würden gar kein Fehlverhalten darstellen. Das Vorgehen der Demokraten gleiche damit einem Coup, und dementsprechend seien die Angriffe auf Trump der eigentliche Skandal und ein Angriff auf die amerikanische Nation.

Spätestens mit den öffentlichen Anhörungen vom November befand man sich dann mitten im Outside-Game: Während das Inside-Game die politischen Auseinandersetzung in den Parlamenten (und Regierungsbehörden) bezeichnet, gestalten im Outside-Game solche Akteure die Konfliktführung, die eigentlich „nur“ Beobachter sind - inzwischen übernehmen die so genannten surrogates als „geliehene“, aber keineswegs unabhängige Expertenstimmen einen großen Anteil in der politischen Berichterstattung. Dass sich dabei auch im Fernsehen mindestens zwei Welten öffnen, überraschte so recht niemanden mehr: Am Tag der ersten Anhörung eröffnete Rachel Maddow ihr Prime-Time-Format bei MSNBC mit den Worten, Trump sei bei etwas „Illegalem erwischt worden“. Sean Hannity von Fox hingegen begrüßte seine Zuschauer zeitgleich mit den Worten, es sei ein ,großer Tag für die Vereinigten Staaten, für das Land, für den Präsidenten - und ein lausiger für die korrupten, nichtsnutzigen, radikalen, extremen, sozialistischen Demokraten und ihre Verbündeten, besser bekannt als der Medien-Mob“ (Grynbaum 2019).

Interessanterweise fassten die bei den Anhörungen beteiligten Republikaner also Insider - die befragten Diplomaten recht „sanft“ an; sie konzentrierten sich darauf, deren Aussagen als „Hörensagen“ abzutun. Weit weniger zurückhaltend zeigte sich Fox: Dort ging man z. B. Oberst Vindman massiv an. „Experten“ und Moderatoren zweifelten offen an seinem Patriotismus, ja unterstellten Spionage. Trump spiegelte das und nannte Vindman auf Twitter wiederholt einen „Never Trumper“, was wohl schlecht sei. Derart machte Fox News einen wichtigen 
Unterschied zum Impeachment von Richard Nixon. Auch der hatte mediale Stützen. Aber seinerzeit waren die Nachrichtenmedien von weit geringerer Reichweite, nicht eingebunden in ein sich gegenseitig ewig weiterzitierendes, rechtskonservatives Medien-Ökoksystem (Benkler et al. 2018).

Konsequenterweise schloss sich Trump, der auf ein Geben-und-Nehmen mit Fox schon lange eingestellt war, in der ersten Woche dem Outside-Game an. Eine der wohl spektakulärsten Befragungen dürfte die von Marie Yovanovitch gewesen sein, die in einer fast sechsstündigen Befragung in klaren Worten schilderte, wie Trump, Giuliani und deren Mitstreiter sie über eine Rufmordkampagne einzuschüchtern versucht hatten, bevor der Präsident sie schließlich ihres Amtes als US-Botschafterin in der Ukraine enthob. Schon zuvor hatte Trumps Sohn Yovanovitch auf Twitter als „Witzfigur“ bezeichnet; nun, während (!) der Befragung, verunglimpftet sie der Präsident höchstselbst live - unwürdiger, mag man meinen, ist schwer vorstellbar.

\section{Das Impeachment als Hörspiel: Podcasts zur Amtsenthebung}

„Heute ist Donnerstag, der 16. Januar - Tag 114 seit der Einleitung der Impeachment-Untersuchung im Repräsentantenhaus." So begann Hayes Brown seinen Podcast Impeachment Today, den das Medienportal BuzzFeed gemeinsam mit der New Yorker Streaming-Plattform iHeartRadio betreibt. Das etwa 20 Minuten lange Hörstück war die 50. Folge des Informationsformates und der Moderator verkündete stolz, genau diese Episode würde in den nächsten Tagen die Download-Zahlen des Podcasts in den siebenstelligen Bereich heben: Die Millionen-Schallgrenze stünde unmittelbar bevor. Danach veränderte sich die Eröffnungssequenz leicht - Brown ergänzte seitdem auch die Verhandlungstage des Senats.

Impeachment Today war so etwas wie das ,junge Format“ einer ganzen Flotte von Ein-Themen-Podcasts, die seit dem vergangenen Herbst das ohnehin schon lebhafte Sortiment politik- und nachrichtenorientierter Hörangebote in den USA ergänzt und erweitert hatten. Hayes Brown beschrieb die Vorgänge im Kongress recht niederschwellig und bemühte sich bei den Interviews um eine verständliche, zugängliche Sprache. In unregelmäßiger Folge traten Gäste aus dem Washingtoner Politikbetrieb in der Sendung auf und meldeten sich zu Wort - dabei waren Journalisten, Wissenschaftler und nicht selten die surrogates aus beiden Lagern. Rubriken wie This fuckin' guy stellten das handelnde Personal näher vor, außerdem vermeldete der Podcast die Stimmungslage in Washington 
auf einer Skala von 0 („,Normaler Tag in Washington“) bis 10 („Richard Nixon tritt zurück und verlässt Washington mit dem Marine One-Helikopter"). Die Werte pendelten meist zwischen 7,5 und 8,0.

Als großer, vernünftiger und nachdenklicher Bruder kam Impeachment, explained von Ezra Klein für den digitalen Medienkonzern Vox daher. Die Folgen dieses Podcasts hatten eine Länge von etwa 60 Minuten und deckten sowohl tagespolitische Entwicklungen wie auch historische Hintergründe ab. Eine solche Langform gilt als deep dive podcast, der viele Details und Expertenwissen vermitteln soll. Auch Klein investierte viel Zeit in die minutiöse Beschreibung des Verfahrens im Kongress und sprach dazu mit Sachverständigen aus Politik, Journalismus und Wissenschaft. Der Podcast wirkte wie ein seriös produziertes Radio-Feature und verfügte über relativ ausführliche Show Notes mit Informationen zu den Inhalten und Gästen jeder Folge.

Selbstverständlich hat auch die renommierte Washington Post in den vergangenen Jahren ein umfangreiches Audio-Angebot aufgebaut. Der schlicht Impeachment betitelte Podcast war dabei eine Art „Aggregator“, der Passagen aus verschiedenen Angeboten gesammelt und täglich bereitgestellt hat. Ergänzt um ähnliche Podcasts von $C N N$ oder $N B C$ erhielt man zwischen Oktober 2019 bis zum Ende des Verfahrens im Februar 2020 ein umfangreiches, mehrmals in der Woche erweitertes Audio-Archiv zum Verlauf des Impeachment-Verfahrens. Die Amtsenthebung als Hörspiel.

\section{Podcasts als Nachrichten im Serienformat}

Im Journalismus scheint der Podcast-Boom ungebrochen und erlaubt Medienakteuren, ihr Portfolio zu ergänzen. Hierzulande hat gerade erst die FAZ mit großem Brimborium den Deutschland-Podcast gestartet - unter etablierten Medienhäusern ist das eher die Nachzügler-Position, im internationalen Vergleich sowieso. Während in traditionellen Hörfunk-Angeboten meist relativ strenge Form- und Formatbegrenzungen gelten, eröffnen die digitalen Hörstücke Experimentierräume. Ein schönes Beispiel jenseits der Impeachment-Podcasts liefert die Wochenzeitung Die Zeit: der Interviewpodcast Alles gesagt ist erst dann vorbei, wenn der Gast nicht mehr weiterreden möchte. Das führt zu Marathonsendungen von mehreren Stunden Dauer. Rekordhalter ist aktuell der YouTuber Rezo mit $8 \mathrm{~h}$ und 40 min Redezeit.

Die Impeachment-Podcasts griffen dabei gleich mehrere Trends auf: Etwa nutzten sie die immer besser werdende technische Ausstattung der Nutzer, die mit ihren Smartphones über leistungsfähige Speicher- und Abspielgeräte 
verfügen und deren Flatrates die Audio-Dokumente gut verkraften können. Pendler- und Wartezeiten können so mit reichlich Inhalt gefüllt werden; auch für das regelmäßige Workout gibt es auditive Begleitung. Außerdem erfolgt die Bereitstellung der Podcasts im Modus der „Serialisierung“, die aus den Mediatheken der Fernsehsender und vor allem durch Streaming-Plattformen bekannt und populär geworden ist. Auch das Podcast-Publikum kann einzelne Beiträge direkt nach Erscheinen konsumieren oder nach Bedarf Beiträge sammeln, um dann in einem Binge-Listening mehrere Folgen hintereinander durchzuhören. In Deutschland hat mit dem Beginn der Coronakrise das vom Norddeutschen Rundfunk produzierte „Coronavirus-Update“ mit dem Berliner Virologen Dr. Christian Drosten eine Art „Kultstatus“ erlangt und viele Nachahmer gefunden.

Den Impeachment-Podcasts fiel es zudem leicht, an fiktionale Polit-Serienwelten wie etwa The West Wing, House of Cards oder Veep anzuknüpfen: Durch die Verfahrensregeln gab es einen klar umrissenen Zeitraum, für die Abläufe im Senat waren ziemlich genau zwei Wochen veranschlagt. Die Demokraten hatten zu Beginn drei Tage Zeit für ihre Eröffnungsplädoyers (22.-24. Januar), danach folgten ebenfalls drei Tage, die für die Republikaner reserviert waren (25., 27. und 28. Januar, an den Sonntagen ruhte das Verfahren). Danach folgte die Gelegenheit zur Befragung, die für die Senator*innen jedoch nur schriftlich möglich war. Die Podcast-Programmierung folgte diesen Vorgaben und mit entsprechenden Vor- und Nachberichten - mit wachsender Intensität bis zum Höhepunkt des Verfahrens, der Abstimmung über die Amtsenthebung am 5. Februar.

Praktischerweise gab es einen recht festen, übersichtlichen Cast of Characters, der in den Lagern die Handlung vorangetrieben hatte. Die Demokratin Nancy Pelosi organisierte als Speaker of the House die Anklage des Präsidenten im Repräsentantenhaus, im Senat trat ihr der republikanische Mehrheitsführer Mitch McConnell entgegen. Pelosi hatte neben der Formulierung der Anklageschrift auch das Team der Impeachment Managers zusammengestellt, die die Klage als Anwälte des Repräsentantenhauses in der zweiten Kammer des Kongresses vertreten. An der Spitze dieses Teams stand Adam Schiff, der im House zuvor die Impeachment Hearings geleitet hatte. Parallel hatte Präsident Trump ein Anwaltsteam benannt, das ihn im Kongress verteidigte. Mit dabei waren unter anderem Kenneth Starr, der als Sonderermittler einst im Impeachment-Verfahren gegen Bill Clinton bekannt wurde, und Alan Dershowitz, ein ehemaliger Harvard-Professor, der als Verteidiger so prominenter wie berüchtigter Mandanten (O.J. Simpson, Jeffrey Epstein) aktiv war.

Mitch McConnell, Senator aus Kentucky, war für die formale Durchführung der Anhörung im Senat zuständig - und in dieser Funktion unterstützte er den Präsidenten als eine Art „Verfahrensanwalt“. Im Impeachment-Verfahren obliegt 
es dem Mehrheitsführer im Senat, wesentliche Regeln für die Verhandlung aufzustellen, etwa die Dauer der Eröffnungs-Statements oder den Modus der Befragung. Auch bei der Bestellung von Zeugen oder der Zulassung von Beweismitteln konnte McConnell als wohlwollender Weichensteller des Präsidenten verstanden werden, wenngleich eine lange Liste an Abstimmungen im Senat über die endgültigen Verfahrensregeln entscheidet. Und so gab in den letzten Tagen das Feilschen um den formalen Prozessverlauf einen Vorgeschmack auf die Debatten - in den Impeachment-Podcasts wurde das Gezerre um die Formalia mit verschiedenen O-Tönen abgebildet und vermittelte ein dichtes Bild von der Stimmungslage im Kapitol.

Das Ensemble aus Anklage und Verteidigung gab während des Verfahrens den Takt für die Impeachment-Podcasts vor. So wurden gleich nach dem Start der Verhandlungen O-Töne aus dem Senat zu einem wichtigen Teil der einzelnen Episoden - im Stile eines highlight reel wurden besonders schwere Beschuldigungen oder scharfe Wortgefechte zusammengefasst und mit den kommentierenden Passagen der Podcasts vermischt. Man liegt also ganz richtig, wenn man sich die mediale Begleitung des Impeachment als eine Art Mash-Up aus Anwaltsserie und Sportberichterstattung vorstellt.

Interessanterweise beschränkten sich Podcasts nicht auf eine Rolle als rein auditive Inhaltsvermittlung - nicht selten bildeten sich um erfolgreiche Formate aktive Zuhörergemeinschaften, die eine Anschlusskommunikation ermöglichen. Besonders erfolgreiche Podcasts wie The Daily oder die Lage der Nation sind dazu übergangen, einzelne Episoden vor Publikum einzuspielen und sich so von der allein digitalen Darreichung zu lösen. Auch die Nutzung der Begleitmaterialien (Show Notes) erlauben die Vernetzung der Audio-Inhalte mit weiteren, thematisch verwandten Online-Inhalten. Hier können Podcasts einen Beitrag zum medialen Agenda-Setting leisten, durchaus in korrigierender Funktion, wenn ansonsten wenig diskutierte Themen verhandelt werden. Und tatsächlich liefern die politischen Turbulenzen der Coronakrise das Material für ein Weiterführen der Hör-Angebote - aus Impeachment Today zum Beispiel wird ab Mitte April News O'Clock mit einem aktualisierten Themenspektrum.

\section{Podcasts als „erzählte Politik“}

Zusätzlich zur regelmäßigen Begleitung der aktuellen Ereignisse übernehmen die Podcasts noch eine weitere Aufgabe für die öffentliche Diskussion politischer Themen - das gilt insbesondere bei der Verhandlung über die Zukunft des Präsidenten. In geradezu paradigmatischer Weise erzählen Podcasts einen 
laufenden politischen Prozess und tragen dadurch zu seiner gesellschaftlichen Wahrnehmung und Einordnung bei. Aus der Sicht des noch jungen Zweiges der politischen Erzählforschung unterstützen Podcasts die Konstruktion von Politik im öffentlichen Diskurs. Durch die Einbindung in alltagsnahe Dialogsituationen wurde die hochformelle Sprache der Verhandlungssituation geerdet und für den normalen Sprachgebrauch bereitgestellt. Gerade die auf Vereinfachung und Erklärung angelegten täglichen Podcasts finden hier ihre Funktion als „Erzählmaschinen“.

Allerdings sind in der immer weiter ausdifferenzierenden Podcast-Landschaft auch Stimmen zu hören, die ihre eigene Agenda verfolgen - allen voran setzte hier ein alter Bekannter aus der Trump-Kampagne von 2016 den Ton. Stephen K. Bannons Podcast mit dem bezeichnenden Namen War Room: Impeachment ließ keine Zweifel zu: Es ging hier nicht um eine neutrale Begleitung des Geschehens, sondern um die Unterstützung der ,eigenen“ Leute und die möglichst vollständige Diskreditierung des politischen Gegners. Anschaulich vorgeführt wurde diese Strategie etwa in Episode 126 vom 22. Januar, als der „Bürgermeister Amerikas“ Rudy Giuliani per Telefon zugeschaltet wurde und das Eröffnungsplädoyer von Adam Schiff in Grund und Boden stampfte: „If you don't state a crime, you never start a trial. Obstruction of Congress is not a crime or misdemeanor. Congress must stop inventing things that are impeachable offenses." Der in seiner Rolle als Anwalt des Präsidenten und durch seine Verwicklung in die Ukraine-Affäre mehr als nur befangene Giuliani entwickelte damit ein für das republikanische Lager sehr anschlussfähiges Deutungsmuster, das allerdings mit dem in der Verfassung niedergelegten Verfahren nichts mehr zu tun hatte.

Formal mag das Impeachment des Jahres 2020 noch der letzten Auflage von 1999 ähneln. Und auch wenn das Verfahren gegen Bill Clinton maßgeblich durch einen radikalen Online-Journalisten angestoßen wurde, so gibt es mittlerweile fundamentale Veränderungen im digitalisierten Mediensystem. Dauerbegleitung im Livestream, Echtzeitkommentare in den sozialen Medien und eben auch die Nacherzählung und Umdeutung des Geschehens in den Podcasts vervielfältigen die Möglichkeiten zur Wahrnehmung der Ereignisse im Kapitol. Das vorläufige Ergebnis dieser Entwicklung ist eine laute, vielstimmige, multiperspektive Darstellung des Impeachment-Verfahrens.

\section{Plattform-Politik}

Die mediale Spaltung des Landes durchzieht also nicht mehr nur die TV-Networks, wenngleich dort die Grenzlinien am schärfsten gezogen sind: Während bei Fox und in den konservativen Wellen des Talk Radio Donald Trump als Opfer einer 
von Niederlagen verbitterten demokratischen Parteielite weichgezeichnet wurde, beschrieben $C N N, M S N B C$ und die New York Times den Präsidenten als überforderten Unternehmer seiner Selbst. Und das sind nur die verbliebenen Vertreter eines Medien-Mainstreams, der seine Hochzeiten im vergangenen Jahrtausend hatte. Diese doppelte Perspektive auf das Impeachment hätte man erwarten können; denn sie reflektiert nur die in den letzten Jahren (nicht erst seit der Präsidentschaft Trumps) immer stärker gewordene Polarisierung der Medienlandschaft und der politischen Öffentlichkeit - die Amerikaner folgen jenen Outlets, die eine für sie ,passende“ Meinung unterstützen und sehen, wenn man so will, zwei Verfahren: Zerrbilder einer demokratischen oder aber einer republikanischen Medienelite.

In den Weiten der digital partisan publics aus Blogs, Foren, FacebookGruppen, Twitter-Hashtags und YouTube-Kanälen wurden die Gegensätze zwischen Blau und Rot noch leuchtender ausgemalt und die Gräben zwischen den Parteien noch tiefer geschaufelt.

Fast nebenbei entwickelte sich das Impeachment-Verfahren dabei auch zum Verhaltenstest für Facebook und Twitter. Beide Plattformen sahen sich nach einer Phase der friedlichen Koexistenz einigen Konfrontationen mit dem politischen Washington ausgesetzt. Angesichts der immer wieder aufkeimenden Debatte um die Wirkungen von fake news versuchte Facebook das Image der sorglosen Vertriebsplattform für alle Arten von Falsch- und Desinformation abzuschütteln. Die Einrichtung offener Anzeigen-Archive, das „Ausflaggen“ falscher Nachrichten und der Einsatz externer fact-checking-Einheiten ließen zwar erkennen, dass das Netzwerk seine Position im politischen Nachrichtenstrom inzwischen hinterfragt. Allerdings funktioniert die Verbreitung falscher oder irreführender Inhalte noch immer beängstigend gut.

Twitter als die deutlich kleinere, aber durch die Tiraden des Präsidenten in das Licht der globalen Öffentlichkeit gerückte Plattform, pflegte einen anderen Umgang mit politischer Werbung. Firmen-Chef Jack Dorsey verkündete im Oktober 2019 das Ende sämtlicher politischer Anzeigen auf der Plattform; die neuen Herausforderungen für den öffentlichen Diskurs seien erheblich und schwer kontrollierbar: „Maschinenlernen zur Optimierung von Nachrichtenversand und Micro-Targeting, ungeprüfte und irreführende Informationen, und , deep fakes'. Das alles mit wachsender Geschwindigkeit, von immer besserer Qualität und in einem überwältigenden Maßstab.“ (Dorsey 2019). Die Regulierung von Kampagneninhalten bleibt hier wie in anderen Medienumgebungen ein Dauerthema.

Ein großer Teil der hyper-personalisierten politischen Kommunikation findet inzwischen sowieso in von außen nicht mehr einsehbaren Winkeln sozialer 
Medien statt, als geschlossene Gruppenkommunikation auf Mega-Plattformen oder innerhalb von Messengerdiensten. Dadurch wird der Sound zugleich immer schriller und immer weniger hörbar - denn offen zugänglich sind solche Kommunikationsräume nicht. Jedoch sind das nicht die oft befürchteten Echokammern. Denn noch begegnen sich die Bewohner von dark social-Strukturen durchaus über andere Plattformen und Formate. So waren die lokalen und regionalen TV-Netzwerke der größte Profiteur der Zwischenwahlen von 2018. Sie konnten Sendezeit im Wert von immerhin gut drei Milliarden Dollar für politische Werbung verkaufen. Nur - was haben die Betrachter dieser TV-Spots überwiegend gesehen? Negativkampagnen, attack ads und nicht themenorientierte issue ads. Es gehörte nicht viel dazu, sich auszumalen, welchen Ton die mediale Begleitmusik des Impeachment im Jahr der Präsidentschaftswahl alsbald anschlug. Polarisierung galore.

\section{Impeachment-Öffentlichkeit und Soziale Medien}

Diese Wahrnehmung gilt zumindest für die klassischen Medienöffentlichkeiten - also Zeitung, lineares Fernsehen, Talk-Radio und für weite Teile der Online-Nachrichtenportale. In den seit den Obama-Kampagnen von 2008 und 2012 politisch salonfähig gewordenen social media gestaltet sich der Prozess der Öffentlichkeitsbildung etwas anders. Zwar sind auch hier die professionellen Medienanbieter unterwegs, doch folgen viele Nutzerinnen und Nutzer bei Facebook, Twitter, Instagram oder Youtube dann auch den Kommentaren und Einschätzungen ihrer Freunde und Bekannten. Oder sie orientieren sich an den Aussagen von Influencern, die Meriten und Reichweite nicht aus den alten Medienumgebungen beziehen, sondern sich aus unterschiedlichen Gründen einen digitalen Namen gemacht haben. Im Verbund mit einem sich allmählich ändernden Informationsverhalten der Bevölkerung, die immer stärker auf soziale Medien als Informationsquelle setzt, wird aus dem skizzierten Riss in der Öffentlichkeit eine klaffende Lücke.

Zugleich aber gerät dabei eine für die Begleitung und Analyse politischer Kampagnen enorm wichtige Funktion ein wenig aus dem Blick: Die Übersetzung des politischen Wettbewerbs von einem kommunikativen in ein ökonomisches Ereignis. Denn längst sind die digitalen Plattformen zu einem massiven Anzeigenmarkt geworden, der bereits seit langer Zeit auf Hochtouren läuft. Daher lohnt es, sich das Geschehen dort etwas genauer anzusehen. Denn wenn das Ringen um die öffentliche Meinung zum entscheidenden Faktor im Impeachment-Verfahren werden kann, könnten die Marktbewegungen dort auch 
Hinweise auf die politischen Kräfteverhältnisse liefern. Die Einsichtnahme in die digitale Werbewelt ist nach der heftigen Kritik insbesondere an der Rolle von Facebook etwas leichter geworden: Das Unternehmen hat seit einer Weile den Zugang zur so genannten Ad Library geöffnet. Dort können sich Außenstehende über den Werbemitteleinsatz von Wirtschaft und Politik informieren. Das ist zwar etwas mühsam, oft redundant und langweilig, aber eben doch auch ganz aufschlussreich.

\section{Die Ad Library von Facebook}

Die Anzeigenbibliothek setzt an einem für den politischen Diskurs nicht unerheblichen Problem an. Die Inhalte der Werbeanzeigen sind nie für das gesamte Publikum sichtbar, sondern können dosiert an beliebig kleine Zielgruppen ausgespielt werden - das Wissen um die Präferenzen und Gewohnheiten ihrer Nutzer bildet einen Teil der Geschäftsgrundlage von Plattformen wie Google, Facebook oder Twitter, die ihren Kunden einen kostenpflichtigen Zugang zu ganz unterschiedlichen Zielgruppen eröffnen. Der Politikwissenschaftler Erik Meyer hat sich aktuelle Facetten politischer Digitalisierung zwischen Partizipation und Plattformisierung genauer angesehen. Soziale Medien sind für ihn längst nicht nur ein wachsender Teil zeitgemäßer Öffentlichkeiten, sondern sie fügen sich auch bestens in die Wettbewerbslogik von Wahlkämpfen ein.

„Für politische Werbung haben Facebook und Google durch ihr fein granuliertes Wissen über das Nutzerverhalten eine ideale Umgebung geschaffen: Hier müssen die Kampagnen nicht einmal genau wissen, was respektive wie sie kommunizieren wollen. Sie können es durch exzessives A-/B-Testing mit minimal variierten Botschaften und unterschiedlich gestalteten Kommunikationsobjekten experimentell herausfinden. Und wer über die Daten seiner Anhängerschaft verfügt, lässt sich dazu passende ,Lookalike Audiences“ liefern, um potenzielle Wähler zu adressieren.“ (Meyer 2019)

Mit der Ad Library folgt Facebook dem Ruf nach Transparenz. Er war insbesondere nach der Wahl von 2016 laut geworden, weil damals über die Plattform ausgespielte falsche und manipulative Inhalte Einfluss auf den Wahlausgang gehabt haben können. Das Archiv der digitalen Anzeigen erlaubt nun einen Echtzeit-Blick auf einen Teil des Kampagnengeschehens, der der Allgemeinheit in der Regel verborgen bleibt. Er verrät vieles über die Formen moderner digitaler Wahlkampfführung. Als neues Format der Wahlberichterstattung findet sich nun die Diskussion der von Facebook selbst bereitgestellten Zusammenfassungen, die 
über wesentliche Trends informieren - parallel dazu lohnt aber auch ein selbstständiger Besuch im Datenlager.

Dabei wurde schnell deutlich, wie intensiv die Möglichkeiten zur Ausspielung von Botschaften bei Facebook (oder Instagram und Whatsapp) genutzt werden. Die Suche nach dem Begriff „Impeachment“ lieferte in einer durchschnittlichen Impeachment-Woche im November etwa 12.000 Ergebnisse. Darunter waren sowohl zum Zeitpunkt der Suchanfrage aktive Anzeigen (circa 2000), wie auch gut fünf Mal so viele schon wieder eingestellte Angebote. Allein das zeigte die enorme Dynamik bei der Ausspielung während des laufenden Amtsenthebungsverfahrens und stützt die Annahme einer beständig wiederholten Folge von Versuch und Irrtum als Grundprinzip der Plattformkampagnen. Auch ohne präzisere quantitative Analyse zeigte sich beim Durchscrollen per Augenschein, aus welchem Lager der Großteil der Anzeigen stammte - die beherrschenden visuellen Motive sind Trump, Trump und dann folgte noch Trump. Zwischen dem 29. November und dem 5. Dezember wurden ca. 8900 Anzeigen registriert, für die die 471.186 US\$ gezahlt wurden - von verschiedenen Organisationseinheiten der Wiederwahl-Kampagne des Präsidenten. Zum Vergleich: Anfang April in der Hochphase der Corona-Krisenkommunikation meldete die Ad Library einen Umsatz von 547.031 US\$ im Namen von Donald Trump. Seit Beginn der Aufzeichnungen im Mai 2018 summiert sich das Volumen der Werbeausgaben auf mehr als 36 Mio. US\$.

Die Frontstellung der zwei politischen Lager zeigte sich natürlich auch in der Anzeigenbibliothek. Der zweitgrößte Werbetreibende in Impeachment-Dingen war in der untersuchten Woche die Trump nahestehende Wahlkampforganisation FreedomWorks, die mit gut 350 Anzeigen gegen Nancy Pelosi, gegen den Ausschussvorsitzenden Adam Schiff und gegen ganz allgemein die democratic extremists mobilisierte - gezahlt wurden dafür vergleichsweise schmale 4242 US\$. Erst auf Rang vier der Liste findet sich mit New Direction New Jersey eine regional aktive Gruppe, die gut 190 Trump-kritische Anzeigen geschaltet hatte.

\section{Show me the money}

Nun waren die Facebook-Anzeigen lediglich ein sehr kleinteiliges Element in der medialen Kakophonie, die das Impeachment-Verfahren begleitet. Doch belegten sie sehr gut einen zentralen Mechanismus in US-amerikanischen Kampagnen und gemessen an den digitalen Moden, die Präsidentschaftswahlkämpfe seit 
den 1990er Jahren immer wieder hervorbringen, sind sie gewiss genau der letzte Schrei, der auch im heraufziehenden Wahljahr ausgerufen werden wird. Sieht man sich die Anzeigen des Trump-Lagers etwas genauer an, dann zeigte sich sehr präzise, welche Wechselwirkungen die Amtsenthebungs-Initiative der Demokraten provoziert hat: Fast alle Anzeigen verbanden die laute Kritik an den Demokraten, die ,die Wahlergebnisse von 2016 umkehren“ wollten, mit der Aufforderung zum Spenden für einen sehr zu Unrecht verfolgten Präsidenten.

Und die Spendenkampagne, die als ein Rebound-Effekt des Impeachment entstanden ist, hatte es in sich. Das Time Magazine machte sich die Mühe, die Facebook-Daten zu Beginn der Impeachment-Debatte genauer auszuwerten und mit Informationen zum Fundraising zu verbinden - dabei zeigte sich ein direkter Zusammenhang zwischen erhöhter Investition in Social Media-Anzeigen und dem „Rücklauf“ von Klein- und Kleinstspenden durch eine Vielzahl von Unterstützerinnen und Unterstützern im ganzen Land: „Within 72 hours of Pelosi's announcement, the campaign had raised \$15 million in small-dollar donations. On a typical day, it raises approximately half a million in small-dollar donations those of $\$ 200$ or under. On Oct. 31, the day the House voted to approve rules for the impeachment inquiry, the campaign raised more than $\$ 3$ million, around six times the norm, a senior campaign official tells TIME." (Bennett und Wilson 2019).

Damit war die weitere Route klar. Bei jedem weiteren Schritt, den die Demokraten zur Forcierung des Impeachment-Verfahrens unternahmen, wurden tausende giftiger Facebook-Anzeigen geschaltet, die es in den Kriegskassen des Trump-Gefolges klingeln ließen. Ein Beispiel: Am 5.12., dem Tag, als Nancy Pelosi die offizielle Vorbereitung einer Anklageschrift verkündete, zahlte die Trump-Kampagne 157.936 US\$ für insgesamt 7643 Facebook-Anzeigen, die sich nur marginal voneinander unterschieden und auf je verschiedene Empfängergruppen zielten.

Neben dem Staunen über Daten und Zahlen räumte der Besuch in der Facebook-Datenkammer zugleich auf mit der Einschätzung, Donald Trump würde sich bei der Wahlkampfplanung auf sein gewinnendes Ego verlassen und es ansonsten nicht so genau nehmen mit aktueller Kampagnentechnologie. Die Facebook-Anzeigen dokumentierten nur das Ausmaß des „Hypertargeting“. Weiterer Flurschaden für die politische Öffentlichkeit ergab sich in der Kombination mit den Bemühungen um die Demobilisierung des Gegners.

Und - es gibt ja nicht nur Donald Trump. Der Wochen-Höchstwert für Facebook-Anzeigen Anfang Dezember ging auf das Konto des damals noch aktiven demokratischen primary-Kandidaten Mike Bloomberg, der an einem 
einzigen Tag 162.885 US\$ ausgegeben hatte. Der „Unternehmer, Philantroph und 3-maliger Ex-Bürgermeister von New York" (Selbstdarstellung) kam innerhalb von sieben Tagen auf Ausgaben von 1,2 Mio. US\$ - allein bei Facebook und den angeschlossenen Plattformen. Auch die Demokraten spielen also ein Money Game. Sie müssen es tun, um im zeit-, kräfte- und eben geldverzehrenden Wettbewerb um die Position als Präsidentschaftsanwärter bestehen zu können - was für Bloomberg galt, traf ebenso auf Warren, Sanders und Biden zu. In der Nutzung der sozialen Medien zum Kampf um Aufmerksamkeit und als Umschlagplatz für politische Unterstützung in Form von Spendengeldern zeigte sich der dominante Kreislauf des US-amerikanischen Wahlkampfs: Ausgetauscht werden nicht Themen, Ideen oder gar Argumente, sondern Geld. Und genau das vertieft die ohnehin schon vorhandenen Gräben in der Gesellschaft. Denn je stärker „Beteiligung“ in „Spenden“ übersetzt wird, desto kleiner wird die Zahl der Bürger, die sich einen solchen „Dialog“ mit politischen Akteuren leisten können.

\section{Das Impeachment als Pferderennen?}

Deutlicher als im Impeachment konnte sich die Spaltung der Vereinigten Staaten kaum spiegeln. Donald Trump zu unterstützen, war für viele republikanische Abgeordnete keine Frage der Analyse, sondern Tagesgeschäft im permanenten Wahlkampf: Schließlich wird das gesamte Repräsentantenhaus sowie ein Drittel des Senats im November 2020 neu gewählt.

Nun sind amerikanische Politikerinnen und Politiker daran gewöhnt, dass das Land und sie selbst in den polls, den Meinungsumfragen, permanent „vermessen“ werden. Das kann als „Zwei-Böden-Theorie“ des politischen Handelns gefasst werden. Zum einen geht es um Positionen und Programme, zum anderen aber auch um deren Wahrnehmung im Lichte von Umfragedaten. In den letzten rund sechs Jahrzehnten ist nachgerade eine „MeinungsforschungsIndustrie" entstanden, die mit hoher Frequenz entsprechende Daten erhebt. Auch durch die stetig steigende Geldmenge, die Jahr für Jahr in die Wahlkämpfe gepumpt wird, herrscht kein Mangel an polls - irgendwo müssen die Milliarden von Spenden-Dollars ja hin. Zudem sind Daten im Format des Horse Race Journalism zentral für die Berichterstattung über Wahlkampagnen: die tagtägliche Beschäftigung damit, wer mit wieviel (Prozent-)Punkten gerade vorne liegt.

Natürlich ist auch der Präsident an einer Öffentlichkeitsarbeit und guten approval ratings interessiert. Das betrifft mehr als nur die Legitimation seiner 
Position. Nicht erst mit Donald Trump nutzt das Weiße Haus das standing des Präsidenten in „,der Öffentlichkeit“, um den formell unabhängigen Kongress $\mathrm{zu}$ beeindrucken. Insofern ist eine Orientierung der Akteure in Washington an Umfragewerten, die vermeintlich vox populi Amerikas spiegeln, selbstverständlicher Teil ihres politischen Handelns. Zu Beginn eines Wahljahres war es daher wenig überraschend, wenn die Abgeordneten des Repräsentantenhauses im Zuge der Impeachment-Untersuchung sehr genau beobachteten, was ihre Wahlkreise von dem Geschehen in Washington so hielten.

Zugleich darf als neue Qualität des Kommunikationsklimas in den Vereinigten Staaten festgehalten werden, dass der relative Einfluss von Fakten gegenüber gefühlten Wahrheiten schwindet. Es ist das eine, wenn der präsidentielle Kommunikationsstil einen ungewöhnlich lockeren Umgang mit der Sachtreue pflegt. Das andere, wenn in der Folge sich im Lande (und bei Abgeordneten) die Idee verfestigt, man habe es eigentlich immer mit zwei Wahrheiten zu tun: einer rein technischen und einer, die sich harmonisch fügt mit eigenen Vorstellungen, wie es hätte sein können (oder sollen). Mit der Präsidentschaft Trump geht ein postmoderner Relativismus ,alternativer Fakten“ einher. Rudolph Giuliani z. B. brachte diese Haltung radikal auf den Punkt, als er in Meet the Press argumentierte (zur Zeit der Russland-Untersuchungen), der Präsident sollte auf keinen Fall aussagen nur auf der Grundlage von ,,somebody's version of the truth“. Worauf ein erstaunter Moderator, Chuck Todd, entgegnete: „Truth is truth!" Giuliani wiederum formulierte es daher für die Ewigkeit noch einmal präziser: „No, it isn't truth. Truth isn't truth.“ (Poniewozik 2019, S. 249).

\section{Der Präsident und das Amtsenthebungsverfahren im Spiegel der Polls}

Zweifellos ist Donald Trump eine der umstrittensten Persönlichkeiten der US-Geschichte. Das spiegelt sich auch in seinen Zustimmungsraten. Seit Amtsantritt liegt dieser Wert ohne große Schwankungen bei etwa $40 \%$. Derart niedrig und dazu noch: konstant niedrig lagen solche approvals für einen Präsidenten seit über 40 Jahren nicht (,Trump Begins Third Year“ 2019). Selbst in der aufkeimenden Corona-Krise gelang Trump kein Befreiungsschlag, wie er für Präsidenten in Krisen- oder Kriegszeiten zu beobachten war: einzig Anfang April erreichten die Werte das Niveau der Inauguration - und auch zu Beginn der Präsidentschaft hatten kaum mehr als $45 \%$ der Befragten die Regierungsarbeit von Donald Trump positiv eingeschätzt. Prägend für Trumps Präsidentschaft 
ist, dass sich die Kluft zwischen den politischen Lagern eher vergrößerte. Die Fronten haben sich verhärtet. Während die Demokraten ein Ende seiner Amtszeit geradezu herbeisehnen, lassen sich die Anhänger des Präsidenten durch seine Amtsführung kaum irritieren.

So zeigen Daten einer Umfrage des renommierten PEW Research Center (von Anfang September 2019), dass das Land je nach Parteineigung so gut wie alles, was die US-Politik beschäftigt, unterschiedlich bewertete („In a Politically Polarized Era“ 2019). So waren republikanische Parteigänger zu etwa $31 \%$ der Meinung, man brauche strengere Waffengesetze - gegenüber $86 \%$ der Demokraten. Nur $17 \%$ der Republikaner hielten den Klimawandel für ein größeres Problem, $73 \%$ der Demokraten dagegen schon. Etwa die Hälfte der Demokraten (49\%) glaubte, weiße Amerikaner würden weit mehr als farbige Amerikaner Vorteile in der US-Gesellschaft haben; nur $7 \%$ der Republikaner sahen das ähnlich. Die $P E W$-Umfrage fasste 30 solcher politischer Einstellungsdimensionen zusammen und fand eine durchschnittliche Differenz von 39 Prozentpunkten zwischen den Lagern. Interessanterweise beeinflusste die Parteibindung die verschiedenen Präferenzen und Einstellungen deutlicher als klassische soziodemographische Faktoren wie Geschlecht, Alter, Bildungsgrad, usw.

In dieser parteipolitisch recht starren Situation war es gerade für die Senator*innen der Republikaner wichtig, wie sich die polls im Zuge des Impeachment entwickeln. Dabei zeigten ihnen Momentaufnahmen immer wieder, dass Amerika auch hier gespalten war. Wer Trump und seine Amtsführung ablehnte, favorisierte auch ein Impeachment. Umgekehrt waren $96 \%$ derjenigen, die gegen ein Amtsenthebungsverfahren waren, zufrieden mit dem Präsidenten. Das war wenig überraschend. Interessanterweise jedoch war das Verfahren nicht das zentrale Thema in den USA im Wahljahr 2020 - lediglich etwas weniger als die Hälfte der Befragten (46\%) gab an, das Impeachment sei extrem oder sehr wichtig für den Wahlentscheid. Um das in Perspektive zu setzen: Die beiden Top-Themen waren die Wirtschaft ( $83 \%$ ) und die Gesundheitsversorgung $(80 \%)$. Und - das galt auch für die im November 2020 so wichtigen independents. Die US-Wählerschaft zeigte sich also - und das unterstrichen viele Umfragen - einerseits interessiert am Impeachment, andererseits aber nicht sonderlich „bewegt“ („Trump Impeachment and Removal“ n. d.). Damit schließt sich die Frage an, ob wir es hier mit einer Art wear-out-Effekt zu tun haben: Einem Erschöpfungs-Effekt, verstärkt durch die inzwischen traditionelle Frontstellung der Parteilager. Das können einzelne Umfragen nur vage klären. Insofern bieten sich so genannte polls of polls an: Datensätze, die mehrere Umfragen zusammenführen. 


\section{FiveThirtyEight}

Ein recht bekanntes Beispiel hierfür ist FiveThirtyEight, eine Nachrichtenwebsite, deren Schwerpunkt im statistik-getriebenen Journalismus liegt. Das Team um Nate Silver hat einen interessanten Datensatz veröffentlicht, der einige Dutzend solcher Meinungsumfragen aggregiert, die danach gefragt hatten, ob Donald Trump seines Amtes enthoben werden sollte - beginnend schon mit den Russland-Untersuchungen von Mueller im Frühjahr 2019 (Bycoffe et al. 2020). Anhand dieser Daten lässt sich nachvollziehen, wie die amerikanische Öffentlichkeit eine Enthebung im Zuge der Ermittlungen zunächst mehrheitlich ablehnte: mit etwas über $50 \%$ gegenüber etwas unter $40 \%$. Die Werte blieben sich im Zeitverlauf recht konstant; allein die Veröffentlichung des Mueller-Reports am 18. April erzeugte einen leichten Aufschwung der Meinungen, die ein Verfahren befürworteten. Bis Anfang Mai indes pendelten die Zahlen zurück. Und sie bleiben recht ausgeglichen bei diesem Verhältnis von knapp $50 \%$ vs. knapp $40 \%$ bis in den September. Auch die Anhörung von Mueller im Kongress am 24. Juli änderte daran nichts - im Gegenteil, es war sogar ein leichter Rückgang in der Unterstützung eines Impeachments zu beobachten.

Tatsächlich verschoben sich die Daten aber in dem Augenblick, in dem Nancy Pelosi am 24. September anlässlich der Ukraine-Vorwürfe die Eröffnung einer Untersuchung verkündete. In den nächsten rund zwei Wochen kehrte sich das Verhältnis um, wenngleich nicht wirklich dramatisch: Am 7. Oktober werden knapp unter $50 \%$ notiert, die für ein Verfahren sind, etwas über $40 \%$ dagegen. In den folgenden Wochen bis zum 23. November ebnete sich dieser ,Vorsprung“ der Befürworter sogar etwas ein - dem Tag, als das House Intelligence Committee seine Zeugenbefragungen beendete. Man darf also festhalten, dass Amerika durch die Ukraine-Affäre und die Anhörungen im Repräsentantenhaus zwar ,angeschoben“ wurde in Richtung einer Amtsenthebung von Trump, aber relativ betrachtet nur langsam und offenbar nicht in einer dramatischen Weise, die Abgeordneten und Senatorinnen hätte überwältigen können.

Betrachtet man hingegen die Daten nach Parteilagern, dann ergab sich ein eindeutiges Bild. Über das Jahr hinweg befürworteten etwas weniger als $70 \%$ der Parteigänger der Demokraten eine Anklage Trumps; rund ein Drittel der „Unabhängigen“ sahen das ähnlich - und immerhin zwischen acht und zehn Prozent der befragten Personen, die dem republikanischen Lager zuneigen. Die Eröffnung der Untersuchung im Repräsentantenhaus gab dieser Haltung einen leichten, aber erkennbaren Schub: Unter den Demokraten gab es zum 
Jahresbeginn 2020 rund $83 \%$ Impeachment-Unterstützer. Bei den Unabhängigen liegt der Wert nun bei rund $44 \%$, unter Republikanern bei knapp elf Prozent. Auch hier also zeigte sich ein - wenn auch geringer - Einfluss des Verfahrens an sich.

\section{Impeachment as expected - ein nicht sonderlich spannendes Pferderennen}

Amerika scheint einiges gewohnt, Trump hatte vieles „eingepreist“, wie man an der Börse vielleicht sagen würde: Die Untersuchungen zu seiner möglichen Amtsenthebung bewirkten in der Öffentlichkeit keine erheblichen Meinungsschwankungen - eben ganz so, als seien das doch historische Geschehen und die Vorwürfe keine sonderlich bedeutsamen news. Wenngleich sichtbare Bewegungen im einstelligen Prozente-Bereich auch entscheidend sein können, so war der Amtsenthebungsprozess kein nützliches taktisches tool; es setzte keine größeren Bewegungen der Meinungen und Einstellungen Amerikas gegenüber Donald Trump in Gang.

Noch im März hatte Nancy Pelosi in einem Interview von einem Impeachment abgeraten. Ein solches Vorgehen sei eher dazu geeignet, die Spaltung des Landes weiter fortzuschreiben. Implizit verwies sie damit auf die geringe Wirkmächtigkeit des Impeachment-Verfahrens selbst. Denn eigentlich ist es ein Mechanismus zur parlamentarischen Kontrolle, und gerade die Orientierung auf den Verlauf der öffentlichen Meinungsbildung zum Impeachment könnte das Instrument selbst entwerten - so blickten denn auch viele demokratischen Senator*innen gespannt auf die Umfragen zu ihrer Wiederwahl im November: Hat eine entschlossene Unterstützung des Amtsenthebungsverfahren möglicherweise Folgen für die Wählerstimmung im eigenen Bundesstaat? In ihrer Studie Investigating the President. Congressional Checks on Presidential Power schätzen Douglas L. Kriner und Eric Schickler (2017) die Wirkungen der Untersuchungspraxis eher zurückhaltend ein. Offenbar spielt der Faktor Zeit eine nicht unerhebliche Rolle erst bei einer Dauer von Wochen und Monaten entfalten Anhörungen eine allmähliche Wirkung auf die öffentliche Meinung. Pelosi dürfte also seinerzeit ganz richtig gelegen haben. Nach der Masse der Umfragen haben die rund 30 Stunden Live-Übertragungen der Zeugenaussagen nur wenige Parteigänger der Republikaner vom Anliegen der Demokraten überzeugt. Es gab keine größeren Absetzbewegungen von Trump. Das Lager seiner Befürworter zeigte sich gefestigt. 


\section{Corona ante portas}

Der Blick in die verschiedenen Medienumgebungen und das Suchen nach dem Pulsschlag der öffentlichen Meinung zeigt ein mehrfach gespaltenes Land: Die Polarisierung entlang der Parteigegensätze härtet aus und wird durch den Tonfall des amtierenden Präsidenten, der keinen Hieb gegen den politischen Gegner ausspart, weiter verschärft. Die Frontstellung schreibt sich fort in den partisan media, die aus ihren Sympathien für die eine oder die andere Seite keinerlei Hehl machen. Neben den noch immer wichtigen „Traditionsmedien“ erzählen digitale Angebote in Gestalt von YouTube-Kanälen, Podcasts oder Twitter-Accounts weiter die zwei Geschichten des Impeachments. Digitale Plattformen wie Facebook werden dabei immer stärker in den Finanzkreislauf des Wahlkampfs eingebunden und fungieren als Geldquelle für die Kampagnen. Das Impeachment-Verfahren als zeitlich klar bestimmter Vorgang mit Anfang und Ende hat diese Prozesse wie unter einem Mikroskop sichtbar gemacht und zugleich die Blaupause für den weiteren Verlauf des Wahljahres geliefert. Allerdings birgt die zum Ende des Impeachments bereits im Hintergrund schwelende Coronakrise das Potenzial, als mächtiges Störfeuer zu wirken. Denn der üblicherweise fest gefügte Wahlkalender mit Vorwahlen, Nominierungsparteitagen und TV-Debatten ist bereits jetzt erheblich ins Wanken geraten. 